\title{
Controversies in the Anesthetic Management of the Obese Surgical Patient
}

\author{
Yigal Leykin, Jay B. Brodsky. Springer, 2013. ISBN 978-88-470-2633-9
}

\author{
Albert Moore, MD \\ Received: 22 May 2013/Accepted: 29 May 2013/Published online: 11 June 2013 \\ (C) Canadian Anesthesiologists' Society 2013
}

Despite the obesity epidemic, optimal anesthetic management of the morbidly obese remains unclear. To provide insight into the perioperative care of these complex patients, the editors have compiled the knowledge of international obesity experts for this relevant publication. Each chapter, written by a different set of experts, is presented as a controversy in the management of the morbidly obese. Part I of this five-part book serves as an introduction, with chapters on whether there should special training for managing morbidly obese patients and how to measure obesity and calculate drug dosages. Part II, titled "Preoperative Concerns", includes chapters on preoperative considerations and preparation for the cardiac and respiratory systems, the consequences of diseases (e.g., diabetes, metabolic syndrome, and obstructive sleep apnea), the risk for gastric acid aspiration, and the benefits of preoperative physical therapy. Part III is concerned with the intraoperative management of the morbidly obese, with chapters on monitoring, positioning, airway management, induction of anesthesia, use of supraglottic airways, ventilation strategies, choice of anesthetic agent, fluid management, laparoscopic concerns, and the role of regional anesthesia. Part IV is concerned with postoperative management of the morbidly obese and includes chapters on where the morbidly obese should be cared for after surgery, the question of postoperative opioid therapy, and the role of analgesic adjuncts. Part V is a grouping of miscellaneous controversies which includes chapters on outpatient surgical centres, Cesarean delivery for the morbidly obese patient, and physiologic changes after bariatric surgery.

A. Moore, MD ( $\square)$

Royal Victoria Hospital, Montreal, QC, Canada

e-mail: moore_albert@hotmail.com
The text is published in black and white and includes tables or figures in a few of the chapters, although very few chapters contain photographic images. Each chapter is 2 to 20 pages in length, and each can easily be read in one sitting. All chapters start with an abstract and finish with a conclusion, which is helpful for highlighting the important points in the chapter. There is an adequate index for readers wishing to find specific information. There are very few typographical errors.

At first glance, the use of controversies as the foundation for this book makes sense, as much of the management of morbidly obese patients is controversial. Nevertheless, this organizational structure also makes for repetition of information. For example, the chapter titled "Does Every Morbidly Obese Patient Need a Complete Preoperative Workup?" contains a section on cardiac preparation, and this chapter is followed by a chapter that discusses the full cardiac workup in morbidly obese patients. Also, the chapter titled "What is the Optimal Position for Induction of Anesthesia for a Morbidly Obese Patient?" is followed by a chapter titled "What is the Best Way to Induce General Anesthesia in Obese Patients?", which includes a section on positioning. When read as separate chapters, the duplicated information may be pertinent, but the information becomes repetitive when several chapters are read consecutively. As happens with many controversial issues, recommendations are sometimes based less on the literature and more on practices at the author's institution. This approach provides valuable guidance but remains anecdotal. Also, because some of the topics have sparse literature specific to obesity, information is sometimes taken from studies in the nonobese populations. A notable example is the chapter on the management of anti-hypertensive medication.

As a result of using controversy as the basis for this book, the target audience becomes unclear. Complete 
information is presented for each controversy; however, the structure does not lend itself to an efficient review of considerations regarding the morbidly obese patient. For this reason, the text would not be my first choice to recommend to a trainee who is looking to learn about management of the obese patient. Perhaps a clinician involved in sporadic care of the obese may find certain chapters useful to read in order to answer a clinical question. Certainly, those who wish to read a review of expert opinions and current literature in the field of morbid obesity will find the text useful.

At least three other textbooks focusing on the perioperative care of the obese have recently been published, two of which are also edited by Brodsky. It is beyond the scope of this review to state how this current text differs from, or adds to, the information that has been published in previous texts.

The field of bariatric anesthesia is still in its infancy, and the optimal perioperative management of the morbidly obese patient remains complicated and unclear. The editors themselves state that "in the future some if not many of their recommendations may change as more experience is gained, as more studies are completed, and more data are published..." Realizing that the field of bariatric medicine will undergo many changes, the editors have produced a book that consolidates the current literature and expert opinion pertaining to the perioperative management of the morbidly obese.

Conflicts of interest None declared. 